\title{
Strategies for mobile Internet publishing in the era of global economic downturn \\ Yao Feng
}

Journal of Hunan University, Changsha, Hunan, China

YAOFENG777@163.COM

Key words: The era of global economic downturn; mobile Internet; coping strategies

Abstract: The economic downturn has brought a huge negative impact on China's media industry, but also to see its (but it still has its) own development opportunities. In particular, the mobile Internet, which has been treated as a new media form of media, not only gradually toward the mainstream, but also in the doldrums to find a development opportunity and its unique value. China's mobile Internet media also has its coping strategies to adapt to the pace of the times.

\section{全球经济低迷时代移动互联网出版的应对策略}

\section{姚锋}

关键词: 全球经济低迷时代; 移动互联网; 应对策略

中文摘要: 经济低迷给中国的传媒业带来巨大负面影响, 也使其看到了自身发展的种种机遇。 特别是移动互联网, 这种一直被当作新兴媒体看待的媒体形式, 不但逐步走向主流化, 更是 在低迷中找到了发展契机和自身独特的价值。我国的移动互联网出版也有其适应时代脚步的 应对策略。

\section{1. 引言}

近年来，全球性的经济低迷对中国社会经济发展影响的深度和广度都是巨大的。对于中 国的传媒业来说，在感受到阵阵寒意的同时，却也看到了自身发展的种种机遇。特别是移动 互联网这种一直被当作新兴媒体看待的媒体形式, “在数字出版的大背景下，自出版应运而生， 成为新兴出版模式之一”， 逐步走向主流化，更找到了发展契机和自身独特的价值。

\section{2. 经济低迷为移动互联网带来“破局”机会}

经济低迷时代之前，中国传媒业处于相对平衡的状态，移动互联网与传统媒体由于传播 特性的不同，各自发展着自己的领地，拥有固定的受众群体。但在表面的平静之下，隐藏着 诸多矛盾, 由于体制、机制、政策、观念等影响, 缺乏根本性的解决。经济低迷使得这些问 题尖锐化, 明朗化, 这个非常的时期反而有助于原有矛盾的加速解决。在这个意义上, 经济 低迷和潜在危机给对中国传媒产业的未来发展带来了“破局”的机会。

\section{1 经济低迷导致受众对信息需求的变化}

1姚锋, 男, 1980 年生, 中南大学法学院博士研究生, 湖南大学期刊社编辑。邮箱: YAOFENG777@163.COM 电话: 18874283650 通讯地址: 中国湖南长沙市中南大学法学院一楼,邮编:410000 
首先是获取信息目的的变化。受众获取信息, 其目的除了一般意义的了解国家大事、世 界局势发展，丰富社会知识外，更多的是通过捕捉合理的市场和技术信息、发现商业机会， 实现拓展就业技能, 积极应对经济低迷对个人和企业带来的影响, 通过对信息的观测与分析, 把不利因素降至最低。

其次是信息消费方式的变化。近年来移动互联网的普及使受众对移动互联网的使用优势 有了切身体会, 低成本高信息量相对于传统媒体来说具有更高的性价比, 受众基于对消费成 本控制而更倾向于使用移动互联网获取信息。经济低迷影响严重的地区的人们减少了出行, 更多的选择在家里上网和看电视。美国近期的数字电视、移动互联网和移动视频的市场报告 显示，此类消费在美国已经达到了历史最高水平。传统电视，移动互联网电视和手机电视都 有不同程度增长 ${ }^{2}$ 。这说明, 电视的移动互联网化满足了观众的需求, 而经济低迷下人们减少 出行更多的在家里进行娱乐活动的时事背景, 则更为移动互联网市场的发展创造了良好的契 机。

目的和方式的变化直接导致受众需求的信息内容发生变化。经济低迷中受众更倾向于获 取视野宽泛、行业指导性强、对个体具有针对性的信息, 移动互联网的可无限链接, 搜索标 的准确高效，视频音频文字兼具的特性，极大地满足了受众的这些需求。

\section{2 市场促使传统媒体的数字化转型}

经济低迷给传媒业带来的影响本质上是市场的影响。一方面，从 2014 年伊始，我国新闻 出版总署就开始探索和推动报纸市场退出机制的建立, 经济衰退对弱势媒体的影响将使这一 进程加快。另一方面, 多媒体的操作是非常困难的, 当经济环境恶化时, 一些处于生存压力 之下的边缘媒体将更加倾向于选择和强大媒体的整合。据 CNNIC 统计, 中国的移动互联网用 户已经超过了美国，成为世界上最大的移动互联网用户国家，移动互联网的渗透率已经达到 了 $69 \%$ 。而且, 移动互联网的影响仍在持续, $80 \%$ 以上的中国人认为生活离不开移动互联网。 2017 年 1 月, 中国移动互联网用户达到 7 亿, 普及率达到近 70\%, 甚至可以说, 移动互联网 正式成为主流媒体。目前, 我国的移动互联网迅速扩展到农村, 传播和影响着更多的人。移 动互联网作为一种新型交流方式的代表, 让传统媒体正面临着巨大的压力。在这种情况下, 许多传统媒体开始或加速了数字化转型。

传统媒体的数字化转型不仅给传统媒体带来了度过“寒冬”的暖意, 也为移动互联网在现 有传媒格局被打破后, 争取了更大发展的机会。一方面, 数字化转型为传统媒体提供了更加 灵活、便利的赢利渠道, 另一方面, 有了强大的传统媒体作后盾, 网络媒体在信息的提供上, 理论上都能够得到整个传统媒体的大力支撑, 其庞大的、高水平的记者编辑队伍, 增强了网 络媒体的专业性。传统媒体的加入，大大提高了网络媒体信息的可信度和公信力，

然而数字化转型，并不是简单地建一个网站或者简单意义上的新旧媒体整合，而是要真 正适应移动互联网的传播规律, 把自己的价值最大化。传统媒体所建立的许多媒体网站实际 上是毫无价值的, 甚至成为媒体的负担。其中一些强势的传统媒体对有价值的移动互联网出 版的并购整合将是一个新的趋势 ${ }^{(3)}$ 。

\section{3 移动互联网行业内部调整凸显马太效应}

在社会资源和资讯流的产生、传递和利用过程中，常常表现出明显的核心趋势和集中取 向, 马太效应在媒体中产生的集中趋势, 使资源和资讯集中, 重点突出, 浪费减少, 形成媒 体间的竞争机制, 最终往往导致强者愈强, 弱者愈弱直至消失。在整体影响力持续提升下, 移动互联网行业内部在经济低迷中同样也产生了震荡调整。经济低迷对移动互联网的发展虽 然也会带来冲击, 但有助于去除泡沫化, 使得其发展更加务实, 对传播效果和经营模式的探 索更加深入。这最终形成了行业内部的优胜劣汰。 
面对宏观经济的不景气, 拥有大量受众和高品质针对性强的强势传播平台, 无疑是一种 优势。例如: 由于腾讯巨大的用户群及具有黏性的社区, 其在品牌广告市场获得更多的份额 ${ }^{4}$ 。 这些都增强了其度过经济低迷严冬的能力。

反观一些竞争能力较弱的网络媒体, 由于对长期以来累计的各种弊端而缺乏创新能力和 受众忠诚度, 在经济低迷来临时几乎没有应对能力, 结果只能被淘汰。在经济低迷中, 移动 互联网内部将留下一批品牌力、影响力、传播力和渗透力都得到进一步提升的强势媒体，而 没有竞争力的弱势媒体最终消失。虽然这种优胜劣汰在移动互联网出版的数量在数量上减少 了，但留下了有竞争力的精英。这对于移动互联网行业整体的发展无疑是有益的。

\section{3. 全球经济低迷时代移动互联网出版的应对策略}

面对经济低迷所带来的挑战与机遇, 移动互联网出版采取了积极主动的变化以应对。可 以说, 在经济逐步复苏的同时, 移动互联网也在逐步发展、变革。

\section{1 数字技术的更深层次应用}

数字技术早已借助移动互联网已渗透到受众生活、工作、学习与娱乐、消费等各个环节, 其强大功能也为人们所认识, 它能实现传播准则对其目标受众全程、多点、精准、不间断式 的接触、沟通与影响, 在经济低迷的环境中, 这样的沟通是所有传播者都乐于看到的结果。 但在经济低迷中, 数字技术功能的强大早已不是最重要的, 关注用户体验才是其通往更深层 次的不二法门。

如前所述，经济低迷时期受众对信息获取的目的、方式和内容形式都发生了变化，这变 化最显著的就是受众更加关注信息的个人化使用。而数字技术发展到现在, 功能愈加强大, 技术堪称高精尖。殊不知对受众来说，他们并不关心一项技术有多高，而只关心这项技术能 做什么, 能带给他们什么方便。许多技术所带来的功能其实并不好, 有些甚至是永远都用不 上的, 这无疑造成了用户使用的不便, 也增加了使用成本。并不是不断研发才能拓展数字技 术，在现有基础上开发能不断满足受众需求的功能，才更为重要。

例如网络视频, 在目前经济不景气的环境下确实受到巨大的冲击。但一些视频网站苦练 “内功”, 在危机中发掘新机遇, 推出网络视频会议业务, 一下子受到众多企业的青崃, 都是 在危机的洗礼中“应运而生”的 。对于视频技术来说, 并没有开发更高级的技术以应对危机, 而是在现有技术的基础上开发出针对性更强的功能与业务, 这其实也是数字技术的深层次发 展。

\section{2 智能化信息的提供}

所谓的智能化信息，用谷歌副总裁李开复的话来说，就是与以往的人找信息不同，实现 了信息找人。也就是说移动互联网的海量信息承载与检索能力, 对受众信息进行持续化的积 累与分析, 并在此基础上有效转化成对其行为与意图的洞察理解, 以主动提供与其意图相匹 配的信息，而不需要受众多次、重复地缩小或更改范围来获取。

在经济低迷时期, 受众对信息搜索的要求更迅速精确, 因此移动互联网出版中出现了许 多优化措施, 以实现网上“信息找人”的主动信息服务。智能信息具有更高的内容意识、无缝 的加密和过滤, 因此服务质量更高。本文建立的智能信息网络是一种基础设施, 它可以在由 许多网络组成的系统中传输数据、语音和视频。具体来说,网络基础设施和安全的智能网络, 无线, 存储和 IP 电话和其他先进的技术集成在一起,作用的复杂的网络系统, 形成一个统一完整 的多层结构的每个部分将超过它的功能, 没有每个组件的新功能。同时, 智能网络是灵活的, 具有自适应网络基础设施可以自动优化, 创建自动预防和自动恢复网络, 并能有效地分配系 统资源。为了满足这些需求，技术必须支持多种特性 ${ }^{\circledR}$ 。 


\section{3 承载更多的社会责任}

进入移动互联网时代，大到国计民生的信息发布，小到日用商品的价格波动都注定了大 众对信息密切的关切程度。当信息流通不畅或不足以满足人们对当前局势的认识的时候, 都 会碰触到受众敏感的神经, 使人们产生心理波动, 进而形成负面社会影响。无论如何基于覆 盖的广泛性和文化属性上产生的巨大社会影响力, 移动互联网都要责无旁贷地要担当起环境 监视的道义, 促进受众对经济低迷的冷静思考。同时, 在传播过程中, 媒体又会针对信息自 觉或不自觉地替受众做出解释与判断, 一些媒介关于经济低迷的报道和分析, 已经打上了国 家意识的烙印。国家通过在这种传播方式上的调控和引导, 帮助人们在当前动荡的国际经济 形势下作出合理反应, 以稳定市场不安的气氛。

\section{4. 全球经济低迷时代的传播变革}

从整个媒体格局来看, 经济低迷无疑是一场大震荡, 给移动互联网带来了机遇和变化, 进而影响了整个中国传媒格局。可以预见, 经济低迷过后传播秩序会发生一系列的改变。

\section{1 主流与非主流的分合转化}

一直以来在媒体中就存在主流与非主流之分。随着移动互联网技术在我国迅猛发展, 网 络媒体在人们的各项生活中发挥着越来越重要和关键的作用, 也给予了更多的便利, 新媒体 开始向传统媒体的既有优势发起挑战。例如, 中央电视台作为中国电视的第一播出平台, 拥 有国内其他媒体所难以比拟的资源和地位。“春晚”享有“新民俗”之誉，是中国娱乐文化的顶 峰, 但在 2015 年的春节前后却受“移动网络春晚”挑战。这也反映了传统主流媒体面临的现实 处境。

经济低迷过后，伴随着受众对信息需求的变化，传统媒体的内容优势和阅读习惯优势已 不复存在, 而传统媒体的数字化转型又使其牌照优势消失, 网络媒体越来越重视社会责任感, 使其公信力大增, 传统主流媒体的种种优势被消解归并于移动互联网为代表的新媒体中了, 现在的所谓主流与非主流的划分已不适用于当前传播格局, 主流与非主流的分合转化逐渐成 为一种不可避免的趋势。

\section{2 移动互联网与其他经济行业结合更加密切}

从传媒生态的角度来看, 经过这场经济低迷, 使移动互联网和其他经济行业都更清楚地 看到了彼此在生存发展中命运休戚相关。

一方面, 移动互联网创新对促进经济发展有积极的促进作用。一种中国的形式可以帮助 经济从更广泛的发展到集约化的工作，两种可以使我们的产业结构更加合理，提高信息产业 在整个经济中的比重, 加快传统产业渗透的力度和速度。这是因为移动互联网不仅降低了专 家的成本, 而且, 从更高的层面来说, 危机是一个共同的话题, 它可以在互联网公司和传统 制造企业之间移动, 以充分理解、对话和分享机会。因此, 在危机之后, 移动互联网不仅将 对消费者水平产生越来越重要的影响, 还将对企业水平产生越来越重要的影响。因此要保持 移动互联网发展与实体经济发展协调, 调动移动互联网上下产业链互动。

另一方面实体经济对移动互联网的发展也应该引起重视。可以说, 充分认识到移动互联 网行业与实体经济之间的共存关系, 采取有效的措施将二者结合得更为紧密, 是盘活这两个 行业的双赢策略。

\section{References}

[1] Wang Yan. Analysis of the Self Publishing Mode Based on mobile Internet -- taking time streaming as an example [J]. new media research.2018 (04): 86-87 
[2] Chen Gang: change has begun [J], media, 2008 (9), 6-12

[3] Chen Gang: the breaking strategy of media development in the economic downturn [J], news and writing, 2009 (2), 38-40

[4] Du Ze-zhuang: media advertising Matthew highlights [J], media, 2009 (3), 61-63

[5] Mobile Internet, the crisis of life - interview with the Secretary General of the China Internet association Huang Cheng-qing [EB/OL], http://www.cnii.com.cn/20080623/ca542192.htm

[6] Dongyang (Dong yang) : intelligent network to virtual [N], China Computer News May 18, 2007 